\title{
Primary Cutaneous Anaplastic Large-Cell Lymphoma
}

\author{
Nerissa Moodley Patiswa Nombona Anisa Mosam \\ Nelson R Mandela School of Medicine, Department of Dermatology, University of \\ KwaZulu-Natal, Durban, South Africa
}

\section{Keywords}

Primary cutaneous anaplastic large-cell lymphoma - Peripheral T cell lymphoma - Anaplastic large cell lymphoma

\begin{abstract}
A 35-year-old male presented with a 6-month history of asymptomatic, generalised, selfhealing lesions. On clinical examination, there were diffuse, ulcerated, necrotic papules and nodules with lymphoedema of the face. Histology sections confirmed atypical lymphoid-type cells which appeared round-to-oval with irregular nuclei (horseshoe-shaped). Immunohistochemistry stains were positive for $\mathrm{CD} 30, \mathrm{CD} 3$, and epithelial membrane antigen. The features were in keeping with an anaplastic large-cell lymphoma, $T$ cell type. This transformed into a systemic variant of the disease after the patient had completed chemotherapy.
\end{abstract}

Published by S. Karger AG, Basel

\section{Introduction}

The peripheral T cell lymphomas (PTCLs) are a heterogeneous group of aggressive neoplasms that constitute $<15 \%$ of all non-Hodgkins lymphomas in adults [1]. Primary cutaneous anaplastic large-cell lymphoma (PC-ALCL) is a CD30+ lymphoproliferative disorder of the skin and accounts for $25-30 \%$ of primary cutaneous lymphomas [2]. They present in the skin without systemic involvement at the time of diagnosis and after completion of initial staging [1]. We report the case of a young African male who presented with this rare neoplasm, which, in the South African context, can be confused for cryptococcosis clinically. 
Fig. 1. a, b Ulcerative papules and nodules present on the face with lymphoedema.

Fig. 2. a, b Papules and nodules present on the trunk and back, healing with post-inflammatory hyperpigmentation and scarring.

Fig. 3. Non-cicatricial, patchy annular alopecia of the scalp.
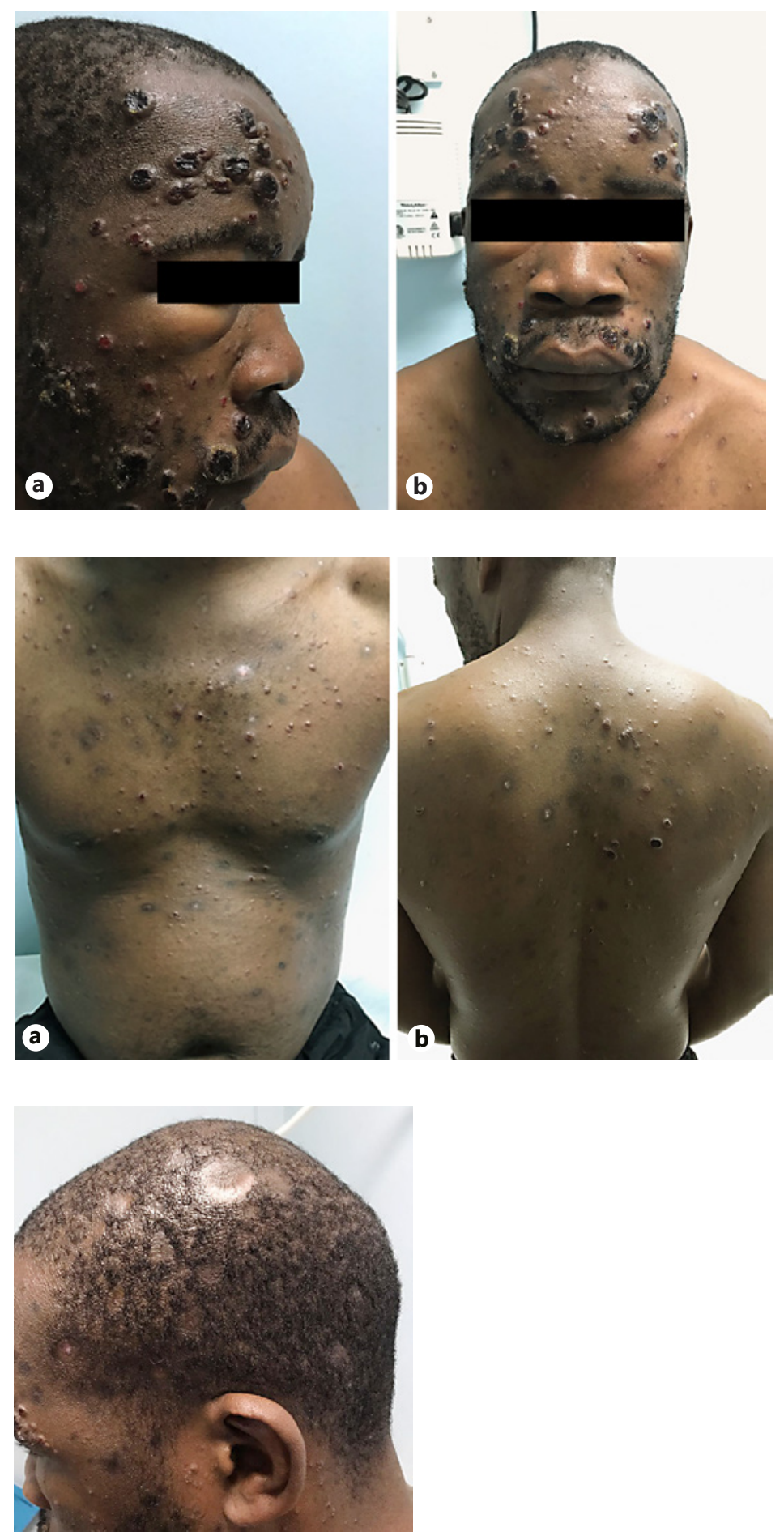

\section{Case Report}

A 35-year-old male from Northern KwaZulu-Natal presented with a 6-month history of generalised, asymptomatic, ulcerated papules and nodules. The lesions had initially started on his trunk and face. They enlarged, became necrotic, and were self-healing. He had associated loss of hair, lethargy, and generalised malaise. 

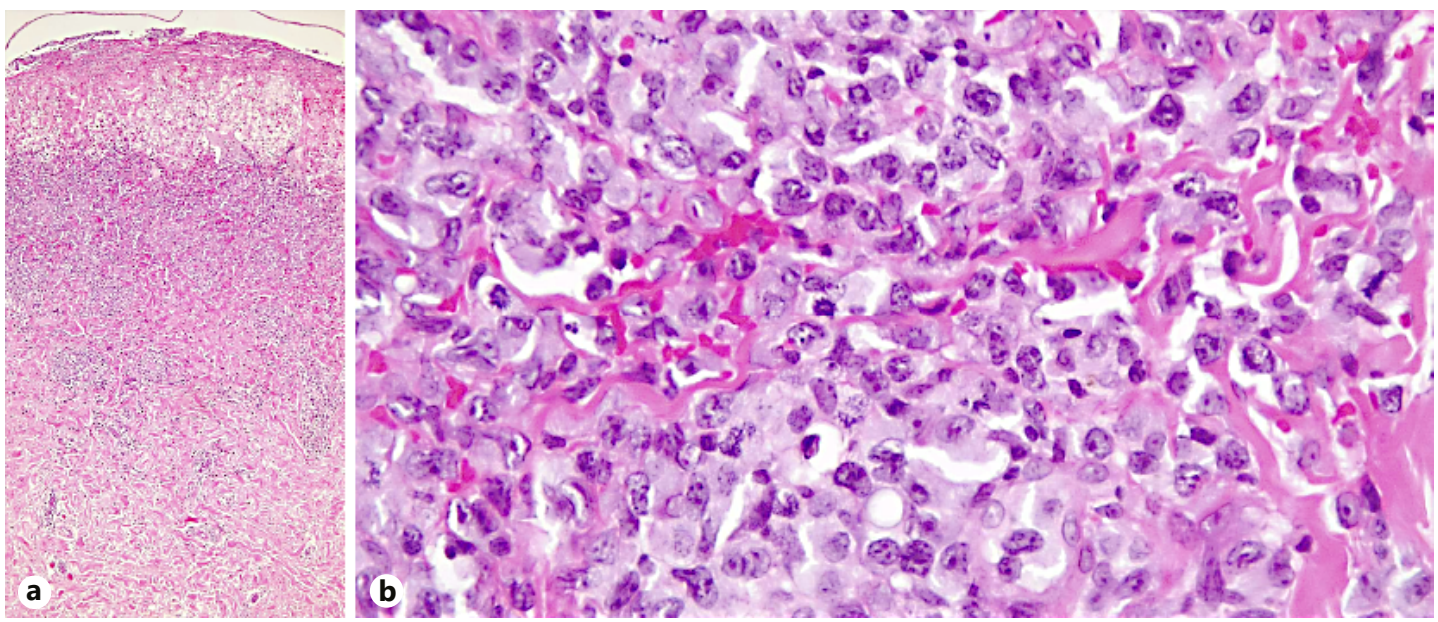

Fig. 4. a Stain showing acanthosis, hyperkeratosis, inflammatory crusting, and spongiosis on low power. H\&E. b Stain showing atypical lymphoid cells which appear round-to-oval, with irregular nuclei (horseshoeshaped). H\&E.

On clinical examination, diffuse, ulcerated, necrotising papules and nodules were noted, with lymphoedema of the face (Fig. 1a, b), and umbilicated papules on the trunk and back (Fig. 2a, b). Non-cicatricial (annular) alopecia was found on the scalp (Fig. 3). There was no palpable lymphadenopathy or hepatosplenomegaly. The rest of the examination was unremarkable. Routine blood parameters were normal. Hepatitis B surface antigen and EpsteinBarr virus IgG were positive. The human immunodeficiency virus (HIV) ELISA was negative.

\section{Results}

Histology sections confirmed atypical, lymphoid-type cells which appeared round-tooval, and with irregular nuclei (Fig. 4a, b). The tumour cells demonstrated a diffuse strong positivity for leukocyte common antigen (LCA) and membranous positivity for CD30, EMA, and CD3 (Fig. 5a-c). There was negativity for AE1/AE3, Melan A, CD20, ALK-1, and CD138. The features were in keeping with ALCL, T cell type. Chest radiography and echocardiogram showed no pathological findings. There was no evidence of infiltration on bone marrow biopsy. Staging computed tomography revealed bilateral inguinal lymphadenopathy (Fig. 6). Based on clinical and histopathological findings, a diagnosis of PC-ALCL was made. The patient was referred to haematology where he received 8 cycles of CHOP (cyclophosphamide, doxorubicin, vincristine, and prednisone). The lesions resolved completely, and a subsequent position emission tomography (PET) scan showed no evidence of metabolically active disease. However, 2 months after completing therapy, the patient relapsed. He presented with generalised nodules on the trunk, face, and upper and lower limbs, with central ulceration (Fig. 7). Further histological examination showed atypical lymphocytes with irregular nuclei which were positive for CD3, CD4, and CD30, and negative for CD2 0 and ALK-1. These features were similar to those described in the first biopsy. A repeat PET scan showed widespread lymph nodes with the largest nodes seen in the pelvis. Assessment revealed a transformation into a systemic variant of the original disease. He was started on interferon- $\alpha$ and filgastrim with a minimal response. Currently, he is on MINE (mesna, ifosfamide, mitoxantrone, and etoposide) chemotherapy until approval of brentuximab vedotin is obtained. He is responding well to treatment. 
Fig. 5. Immunohistochemistry demonstrating positivity for CD30 (a), EMA (b), and CD3 (c).
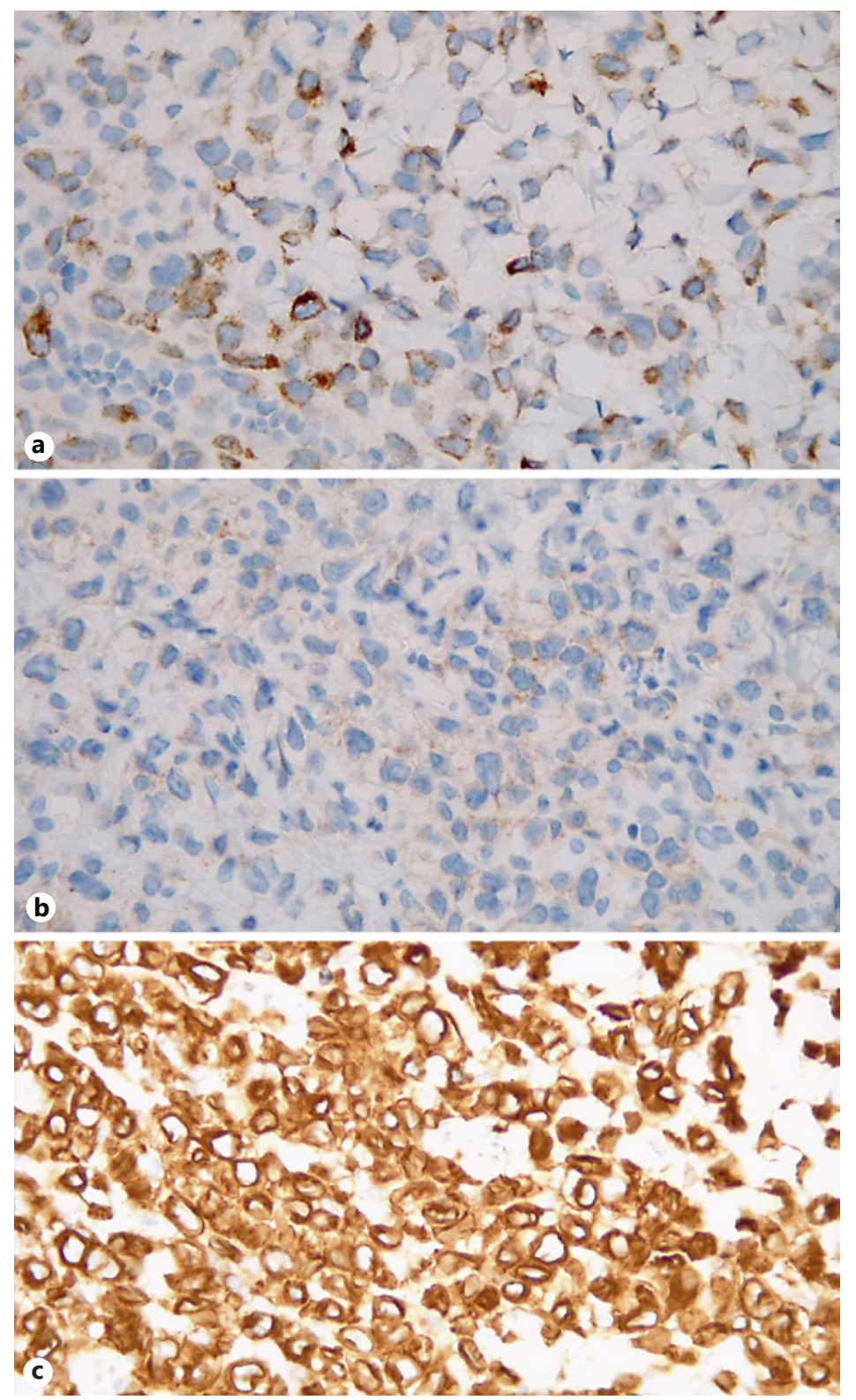

\section{Discussion}

PC-ALCL represents the second-most common clonal T cell neoplasm of the skin after mycosis fungoides [2]. According to the 2016 revision of the World Health Organisation classification of lymphoid neoplasms, it is a lymphoma with indolent clinical behaviour $[1,3]$. The tumour cells are large, with a pleomorphic, anaplastic, or immunoblastic cytomorphology. There is an expression of the CD30 antigen by the majority of the tumour cells [1]. In comparison to systemic ALCL, PC-ALCL lacks the expression of anaplastic lymphoma kinase (ALK) and does not have gene rearrangements involving the $A L K$ gene [2, 4]. This is a rare lymphoma and the exact incidence is unknown. In an analysis of the Surveillance, Epidemiology and End Results (SEER) database conducted in 2008, only 157 cases of primary, localised CD30+ lymphoproliferative disorders were documented over a 30-year 
Fig. 6. Post-contrast-CT images showing bilateral inguinal lymphadenopathy, with the largest lymph node measuring $3.7 \mathrm{~cm}$ (long axis) $\times 1.4 \mathrm{~cm}$ (short axis).

Fig. 7. Recurrence of ulcerated umbilicated nodules on the right hand after completing chemotherapy.

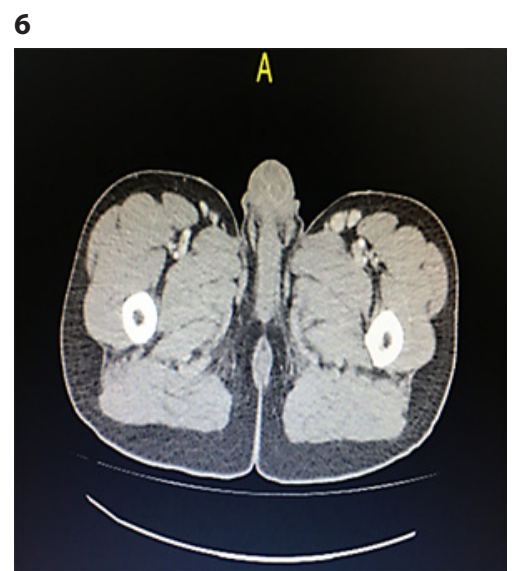

7

period [4]. It mainly affects adults 50-70 years of age and has a male-to-female ratio of 2:1 $[1,2]$. Patients present with asymptomatic, solitary, grouped, or multifocal papules or nodules, usually affecting the upper half of the body $[1,2,5]$. The lesions may persist over weeks to months and measure several centimetres, and the nodules may ulcerate over time [5]. The lesions regress in $50 \%$ of patients, $20 \%$ may present with multifocal lesions, and $10 \%$ will have extracutaneous dissemination $[1,2,5]$. Involvement of regional lymph nodes does not necessarily indicate the presence of systemic disease, as pathologic involvement of local nodes alone does not impact the prognosis in patients with PC-ALCL [6]. Poor prognostic factors include extensive limb disease, an age older than 60 years, and extracutaneous disease, whilst spontaneous regression and an age of under 60 years are favourable prognostic indicators [2].

The aetiology is multifactorial. In normal lymphoid tissue, CD30+ cells are present in a small percentage of patients [2]. These cells are the proposed precursor cells to systemic ALCL. Interaction between CD30 and its ligand (CD30L) is thought to mediate the anti-proliferative and proliferative stages of the disease [2]. Expression of CCR8 and CCR10, the chemokine receptors involved in skin localisation, is also thought to mediate cutaneous presentation. Another hypothesis is that cutaneous T cell lymphomas are the result of chronic $\mathrm{T}$ cell activation by Langerhans cell-mediated antigen presentation [1,2].

There is also an increased expression of toll-like receptors by keratinocytes which play a role in innate immunity [2]. This may be secondary to an infectious insult [2]. Cases of PC-ALCL related to the Epstein-Barr virus [7], Chlamydophila pneumoniae [8], and Human Herpesvirus 8 [8] have been described, but no definite role has been elucidated [2, 7, 8]. HIV also remains a risk factor for ALK-negative ALCL [2].

Histologically, there are dense dermal infiltrates that do not usually involve the epidermis. The infiltrate is comprised of cohesive sheets of large CD30+ tumour cells that morphologically resemble those of systemic ALCL, i.e., "Hallmark" large cells with abundant, pale, eosinophilic cytoplasm, and round or pleomorphic horseshoe-shaped nuclei with prominent nucleoli $[1,2]$. In $25 \%$ of cases, there is an atypical, non-anaplastic Reed-Sternberg-like appearance [1]. Reactive lymphocytes, histiocytes, eosinophils, and neutrophils are commonly 
present in the periphery of the tumour, and, in ulcerating lesions, epidermal hyperplasia may be prominent [1].

The differential diagnosis includes lymphomatoid papulosis (LyP), systemic ALCL, transformed mycosis fungoides, and reactive lymphoid hyperplasia [1]. LyP has a similar morphologic and immunohistochemical appearance to PC-ALCL. Therefore, the correlation of pathological findings with clinical history is essential [2]. In contrast to PC-ALC, LyP is a recurrent, self-healing condition, characterized by a papulonodular skin eruption [1]. Lesions are usually smaller $(<1 \mathrm{~cm})$ and resolve spontaneously within a few weeks or months [1, 2, 9]. A waxing and waning clinical course is consistent with LyP $[1,2,9]$. Pustular lesions or ulcers are rarely seen [9] and extracutaneous disease occurs in only $10 \%$ of cases [10].

In our patient, most of the lesions were $>1 \mathrm{~cm}$ and were ulcerating. Although initially some of the lesions were self-healing, the majority did not regress spontaneously. Therefore, the diagnosis of PC-ALCL was considered in conjunction with the pathology findings. It is quite possible that the patient may have had LyP prior to the diagnosis of PC-ALCL, as LyP may be concurrent, preceded, or followed by another type of cutaneous or systemic lymphoma including ALCL or mycosis fungoides [2, 9-11]. The term "borderline" is used for cases where a distinction between PC-ALCL and LyP cannot be made despite careful clinical and pathological evaluation; Dawn et al. [10] published a unique case illustrating the overlapping of the histological, immunophenotypic, and clinical features of LyP and ALCL.

PC-ALCL is an indolent disease and it is important to avoid overtreatment. Localised lesions can be treated with surgical excision and radiation $[1,2]$. The multiple recurrence of lesions may warrant systemic therapy [1,2]. Patients presenting with widespread lesions may require multi-agent chemotherapy like CHOP $[1,2]$. This, however, has not been proven to be superior to less aggressive agents, and skin relapses following therapy are common [1]. Other systemic treatments include methotrexate, bexarotene (systemic rexinoid), and stemcell transplantation [2]. Brentuximab vedotin (an anti-CD30 monoclonal antibody) is an alternative for relapsed ALCL $[1,2]$.

The exact incidence of PC-ALCL in the South African context is unknown as there is a dearth of reported cases. This report highlights the importance of the early diagnosis and treatment of PC-ALCL. The correlation of clinical findings with histopathology and immunopathology is essential for the management of this disease. Regardless of the good prognosis, it is necessary to closely monitor these patients because of the potential risk of dissemination and extracutaneous spread.

\section{Acknowledgements}

The authors would like to thank the Department of Anatomical Pathology at Inkosi Albert Luthuli Central Hospital for the histopathology reports and images, the Department of Radiology at Stanger Provincial Hospital for the radiographic images, and the Department of Haematology at King Edward VIII Hospital for the updated clinical information.

\section{Statement of Ethics}

The authors have no ethical conflicts to disclose. Informed consent was obtained from the patient. 
Moodley et al.: Primary Anaplastic Large-Cell Lymphoma

\section{Disclosure Statement}

The authors have no conflicts of interest to declare.

\section{Author Contributions}

Dr. N. Moodley contributed her knowledge of the topic, wrote the manuscript, and performed the literature search. Dr. P. Nombona edited the article and approved the final draft. Prof. A Mosam contributed her knowledge of the topic, edited the article, and approved the final draft.

\section{References}

1 Bolognia JL, Schaffer JV, Cerroni L, editors. Dermatology. Volume 2. 4th ed, revised. New York: Elsevier; 2018. pp. 2127-47.

2 Brown RA, Fernandez-Pol S, Kim J. Primary cutaneous anaplastic large cell lymphoma. J Cutan Pathol. 2017 Jun;44(6):570-7.

3 Swerdlow SH, Campo E, Pileri SA, Harris NL, Stein H, Siebert R, et al. The 2016 revision of the World Health Organization classification of lymphoid neoplasms. Blood. 2016 May;127(20):2375-90.

4 Yu JB, Blitzblau RC, Decker RH, Housman DM, Wilson LD. Analysis of primary CD30+ cutaneous lymphoproliferative disease and survival from the Surveillance, Epidemiology, and End Results database. J Clin Oncol. 2008 Mar;26(9):1483-8.

5 Oliveira LS, Nobrega MP, Monteiro MG, Almeida WL. Primary cutaneous anaplastic large-cell lymphoma - case report. An Bras Dermatol. 2013 Nov-Dec;88(6 Suppl 1):132-5.

6 Bekkenk MW, Geelen FA, van Voorst Vader PC, Heule F, Geerts ML, van Vloten WA, et al. Primary and secondary cutaneous CD30(+) lymphoproliferative disorders: a report from the Dutch Cutaneous Lymphoma Group on the long-term follow-up data of 219 patients and guidelines for diagnosis and treatment. Blood. 2000 Jun; 95(12):3653-61.

7 Ma L, Katz Y, Sharan KP, Schwarting R, Kim AS. Epstein-Barr virus positive anaplastic large cell lymphoma: myth or reality? Int J Clin Exp Pathol. 2010 Nov;4(1):100-10.

8 Borghi A, Caselli E, Di Luca D, Sebastiani A, Perri P, Seraceni S, et al. Detection of Chlamydophila pneumoniae and human herpesvirus 8 in primary cutaneous anaplastic large-cell lymphoma: a case report. Infect Agent Cancer. 2013 Oct;8(1):41.

9 Kempf W, Kerl K, Mitteldorf C. Cutaneous CD30-positive T-cell lymphoproliferative disorders-clinical and histopathologic features, differential diagnosis, and treatment. Semin Cutan Med Surg. 2018 Mar;37(1):24-9.

10 Dawn G, Morrison A, Morton R, Bilsland D, Jackson R. Co-existent primary cutaneous anaplastic large cell lymphoma and lymphomatoid papulosis. Clin Exp Dermatol. 2003 Nov;28(6):620-4.

11 Solovan C, Baderca F. Lymphomatoid papulosis misdiagnosed as anaplastic lymphoma. J Cytol Histol. 2016;7: 415. 